\title{
ERRATUM
}

Open Access

\section{Erratum to: 'Quality of life and costs of spasticity treatment in German stroke patients'}

Reinhard Rychlik ${ }^{1}$, Fabian Kreimendahl ${ }^{1 *}$, Nicole Schnur ${ }^{3}$, Judith Lambert-Baumann ${ }^{3}$ and Dirk Dressler ${ }^{2}$

Unfortunately, the original version of this article [1] contained errors. There were formatting errors in the main text and in Tables 1, 2, 3, 4, 5 and 6. These tables have been included correctly below. There will also be an update to correct the errors in the main text.

\footnotetext{
* Correspondence: Fabian.Kreimendahl@ifeg.de

${ }^{1}$ Institute of Empirical Health Economics, Am Ziegelfeld 28, D-51399

Burscheid, Germany

Full list of author information is available at the end of the article
} 
Table 1 Cost data sources

\begin{tabular}{|c|c|}
\hline Item & Cost sources \\
\hline Drugs & German Rote Liste 2013, web-based research \\
\hline $\begin{array}{l}\text { Ambulatory medical } \\
\text { treatment }\end{array}$ & $\begin{array}{l}\text { German value measurement (EBM 2000+) } \\
\text { and fee regulations for doctors (GOÄ) }\end{array}$ \\
\hline $\begin{array}{l}\text { Non-pharmacological } \\
\text { therapies }\end{array}$ & $\begin{array}{l}\text { According to agreements between } \\
\text { German health insurance funds and } \\
\text { professional organizations }\end{array}$ \\
\hline Medical devices / aids & Web-based research \\
\hline $\begin{array}{l}\text { Hospitalisation and } \\
\text { Rehabilitation }\end{array}$ & $\begin{array}{l}\text { German Diagnosis-Related Groups (G-DRG), } \\
\text { web- and phone-based research }\end{array}$ \\
\hline Nursing home care & $\begin{array}{l}\text { According to German long term } \\
\text { care insurance }\end{array}$ \\
\hline $\begin{array}{l}\text { Reduction in earning } \\
\text { capacity }\end{array}$ & $\begin{array}{l}\text { Average payments according to German } \\
\text { retirement insurance }\end{array}$ \\
\hline
\end{tabular}

Table 2 Patient demography and other baseline characteristics

\begin{tabular}{|c|c|c|c|c|c|}
\hline & $\begin{array}{l}\text { INCO } \\
\text { pretreated } \\
N=67\end{array}$ & $\begin{array}{l}\text { INCO } \\
\text { naïve } \\
N=41\end{array}$ & $\begin{array}{l}\text { INCO } \\
\text { total } \\
N=108\end{array}$ & $\begin{array}{l}\text { CON } \\
N=110\end{array}$ & $\begin{array}{l}\text { Total } \\
N=218\end{array}$ \\
\hline Gender (m) & $\begin{array}{l}36 \\
(53.7 \%)\end{array}$ & $\begin{array}{l}22 \\
(53.7 \%)\end{array}$ & $\begin{array}{l}58 \\
(53.7 \%)\end{array}$ & $\begin{array}{l}70 \\
(63.6 \%)\end{array}$ & $\begin{array}{l}128 \\
(58.7 \%)\end{array}$ \\
\hline Age (years) & $\begin{array}{l}62.3 \\
(10.7)\end{array}$ & $\begin{array}{l}60.7 \\
(16.0)\end{array}$ & $\begin{array}{l}61.7 \\
(12.9)\end{array}$ & $\begin{array}{l}67.8 \\
(12.7)\end{array}$ & $\begin{array}{l}64.8 \\
(13.1)\end{array}$ \\
\hline $\begin{array}{l}\text { Body mass } \\
\text { index }\left(\mathrm{kg} / \mathrm{m}^{2}\right)\end{array}$ & $\begin{array}{l}26.7 \\
(4.0)\end{array}$ & $\begin{array}{l}26.8 \\
(4.4)\end{array}$ & $\begin{array}{l}26.7 \\
(4.1)\end{array}$ & $\begin{array}{l}27.7 \\
(4.8)\end{array}$ & $\begin{array}{l}27.2 \\
(4.5)\end{array}$ \\
\hline $\begin{array}{l}\text { Time since } \\
\text { apoplex (years) }\end{array}$ & $\begin{array}{l}8.0 \\
(5.6)\end{array}$ & $\begin{array}{l}6.8 \\
(6.1)\end{array}$ & $\begin{array}{l}7.5 \\
(5.8)\end{array}$ & $\begin{array}{l}5.3 \\
(5.1)\end{array}$ & $\begin{array}{l}6.5 \\
(5.6)\end{array}$ \\
\hline $\begin{array}{l}\text { Time since } \\
\text { spasticity (years) }\end{array}$ & $\begin{array}{l}6.9 \\
(6.3)\end{array}$ & $\begin{array}{l}6.0 \\
(6.5)\end{array}$ & $\begin{array}{l}6.6 \\
(6.3)\end{array}$ & $\begin{array}{l}4.9 \\
(5.4)\end{array}$ & $\begin{array}{l}5.7 \\
(5.9)\end{array}$ \\
\hline $\begin{array}{l}\text { Concomitant } \\
\text { diseases (yes) }\end{array}$ & $\begin{array}{l}55 \\
(82.1 \%)\end{array}$ & $\begin{array}{l}29 \\
(70.7 \%)\end{array}$ & $\begin{array}{l}84 \\
(77.8 \%)\end{array}$ & $\begin{array}{l}96 \\
(87.3 \%)\end{array}$ & $\begin{array}{l}180 \\
(8.6 \%)\end{array}$ \\
\hline $\begin{array}{l}\text { Employed } \\
\text { (yes) }\end{array}$ & $\begin{array}{l}1 \\
(1.5 \%)\end{array}$ & $\begin{array}{l}3 \\
(7.3 \%)\end{array}$ & $\begin{array}{l}4 \\
(3.7 \%)\end{array}$ & $\begin{array}{l}9 \\
(8.2 \%)\end{array}$ & $\begin{array}{l}13 \\
(6.0 \%)\end{array}$ \\
\hline $\begin{array}{l}\text { Retired } \\
\text { (yes) }\end{array}$ & $\begin{array}{l}58 \\
(96.7 \%)\end{array}$ & $\begin{array}{l}39 \\
(97.5 \%)\end{array}$ & $\begin{array}{l}87 \\
(94.6 \%)\end{array}$ & $\begin{array}{l}86 \\
(86.9 \%)\end{array}$ & $\begin{array}{l}173 \\
(90.6 \%)\end{array}$ \\
\hline $\begin{array}{l}\text { Early retirement } \\
\text { due to } \\
\text { spasticity (yes) }\end{array}$ & $\begin{array}{l}40 \\
(63.5 \%)\end{array}$ & $\begin{array}{l}20 \\
(55.6 \%)\end{array}$ & $\begin{array}{l}60 \\
(60.6 \%)\end{array}$ & $\begin{array}{l}19 \\
(20.4 \%)\end{array}$ & $\begin{array}{l}79 \\
(41.1 \%)\end{array}$ \\
\hline $\begin{array}{l}\text { Reduction in earning } \\
\text { capacity due to } \\
\text { spasticity (yes) }\end{array}$ & $\begin{array}{l}22 \\
(32.9 \%)\end{array}$ & $\begin{array}{l}11 \\
(26.8 \%)\end{array}$ & $\begin{array}{l}33 \\
(30.6 \%)\end{array}$ & $\begin{array}{l}23 \\
(20.9 \%)\end{array}$ & $\begin{array}{l}56 \\
(25.7 \%)\end{array}$ \\
\hline $\begin{array}{l}\text { Level of } \\
\text { care (none) }\end{array}$ & $\begin{array}{l}12 \\
(17.9 \%)\end{array}$ & $\begin{array}{l}10 \\
(24.4 \%)\end{array}$ & $\begin{array}{l}22 \\
(20.4 \%)\end{array}$ & $\begin{array}{l}37 \\
(35.6 \%)\end{array}$ & $\begin{array}{l}59 \\
(27.8 \%)\end{array}$ \\
\hline Level 1 & $\begin{array}{l}28 \\
(41.8 \%)\end{array}$ & $\begin{array}{l}15 \\
(36.6 \%)\end{array}$ & $\begin{array}{l}43 \\
(39.8 \%)\end{array}$ & $\begin{array}{l}33 \\
(31.7 \%)\end{array}$ & $\begin{array}{l}76 \\
(35.8 \%)\end{array}$ \\
\hline Level 2 & $\begin{array}{l}23 \\
(34.3 \%)\end{array}$ & $\begin{array}{l}15 \\
(36.6 \%)\end{array}$ & $\begin{array}{l}38 \\
(35.2 \%)\end{array}$ & $\begin{array}{l}29 \\
(27.9 \%)\end{array}$ & $\begin{array}{l}67 \\
(31.6 \%)\end{array}$ \\
\hline Level 3 & $\begin{array}{l}4 \\
(6.0 \%)\end{array}$ & $\begin{array}{l}1 \\
(2.4 \%)\end{array}$ & $\begin{array}{l}5 \\
(4.6 \%)\end{array}$ & $\begin{array}{l}5 \\
(4.8 \%)\end{array}$ & $\begin{array}{l}10 \\
(4.7 \%)\end{array}$ \\
\hline
\end{tabular}

All values are means ( \pm standard deviation) or number of patients (\%)
Table 3 Overview of antispastic therapies and measures during the study

IncobotulinumtoxinA group:

Antispastic medications except BoNT/A, non-pharmacological therapies and aids

$\begin{array}{lllll} & \begin{array}{l}\text { First } \\ \text { quarter } \\ (\mathrm{n}=108)\end{array} & \begin{array}{l}\text { Second } \\ \text { quarter } \\ (\mathrm{n}=102)\end{array} & \begin{array}{l}\text { Third } \\ \text { quarter } \\ (\mathrm{n}=99)\end{array} & \begin{array}{l}\text { Fourth } \\ \text { quarter } \\ (\mathrm{n}=94)\end{array} \\ \begin{array}{l}31(28.7 \%) \\ 23(32.4 \%)\end{array} & 20(20.2 \%) & 18(19.1 \%) \\ \begin{array}{l}\text { Oral } \\ \text { medication }\end{array} & 60(55.6 \%) & 54(52.9 \%) & 54(54.5 \%) & 51(54.3 \%) \\ \begin{array}{l}\text { Physical } \\ \text { therapy } \\ \begin{array}{l}\text { Occupational } \\ \text { therapy }\end{array}\end{array} & 43(39.8 \%) & 42(41.2 \%) & 41(41.4 \%) & 44(46.8 \%) \\ \begin{array}{l}\text { Speech } \\ \text { therapy }\end{array} & 10(9.3 \%) & 8(7.8 \%) & 8(8.1 \%) & 9(8.6 \%) \\ \begin{array}{l}\text { Other } \\ \text { therapies }\end{array} & 3(2.8 \%) & 6(6.0 \%) & 4(4.0 \%) & 4(4.3 \%) \\ \begin{array}{l}\text { Therapeutic } \\ \text { aids }\end{array} & 12(11.0 \%) & 5(5.7 \%) & - & 1(1.0 \%)\end{array}$

Conventional therapy group:

Antispastic medications, non-pharmacological therapies and aids

\begin{tabular}{|c|c|c|c|c|}
\hline & $\begin{array}{l}\text { First } \\
\text { quarter } \\
(n=110)\end{array}$ & $\begin{array}{l}\text { Second } \\
\text { quarter } \\
(n=98)\end{array}$ & $\begin{array}{l}\text { Third } \\
\text { quarter } \\
(n=91)\end{array}$ & $\begin{array}{l}\text { Fourth } \\
\text { quarter } \\
(n=84)\end{array}$ \\
\hline $\begin{array}{l}\text { Oral } \\
\text { medication }\end{array}$ & 67 (60.9 \%) & 66 (67.3\%) & 63 (69.2 \%) & $58(69.0 \%)$ \\
\hline $\begin{array}{l}\text { Physical } \\
\text { therapy }\end{array}$ & 68 (61.8\%) & 59 (60.2 \%) & 54 (54.5\%) & 52 (61.9\%) \\
\hline $\begin{array}{l}\text { Occupational } \\
\text { therapy }\end{array}$ & 15 (13.6 \%) & 11 (11.2\%) & $11(12.1 \%)$ & 8 (9.5 \%) \\
\hline $\begin{array}{l}\text { Speech } \\
\text { therapy }\end{array}$ & 5 (4.6\%) & 5 (5.1\%) & 5 (5.5\%) & 4 (4.8\%) \\
\hline $\begin{array}{l}\text { Other } \\
\text { therapies }\end{array}$ & $3(2.7 \%)$ & 5 (5.1\%) & - & $1(1.2 \%)$ \\
\hline $\begin{array}{l}\text { Therapeutic } \\
\text { aids }\end{array}$ & 10 (11.0\%) & 8 (8.2\%) & 12 (13.2 \%) & 8 (9.5 \%) \\
\hline
\end{tabular}


Table 4 Responder analyses at study end after 1-year of treatment

\begin{tabular}{|c|c|c|c|c|c|c|c|}
\hline & INCO pretreated & INCO naïve & INCO total & CON & $\begin{array}{l}\text { INCO pretr. } \\
\text { vs. CON }\end{array}$ & $\begin{array}{l}\text { INCO naïve } \\
\text { vs. CON }\end{array}$ & $\begin{array}{l}\text { INCO tota } \\
\text { vs. CON }\end{array}$ \\
\hline $\begin{array}{l}\text { Shoulder adduction/ } \\
\text { internal rotation }\end{array}$ & 56.4 & 73.9 & 62.9 & 15.5 & $<0.01$ & $<0.01$ & $<0.01$ \\
\hline Shoulder abduction & 65.5 & 100 & 73.0 & 19.7 & $<0.01$ & $<0.01$ & $<0.01$ \\
\hline Shoulder elevation & 66.7 & 88.9 & 72.7 & 20.6 & $<0.01$ & $<0.01$ & $<0.01$ \\
\hline Flexed elbow & 78.3 & 92.9 & 83.8 & 26.9 & $<0.01$ & $<0.01$ & $<0.01$ \\
\hline Forearm pronation & 81.4 & 73.7 & 79.0 & 22.0 & $<0.01$ & $<0.01$ & $<0.01$ \\
\hline Flexed wrist & 82.1 & 94.7 & 86.2 & 26.6 & $<0.01$ & $<0.01$ & $<0.01$ \\
\hline Thumb-in-palm & 77.8 & 81.3 & 78.8 & 20.0 & $<0.01$ & $<0.01$ & $<0.01$ \\
\hline Clenched fist & 79.1 & 95.2 & 84.4 & 22.2 & $<0.01$ & $<0.01$ & $<0.01$ \\
\hline $\begin{array}{l}\text { Intrinsic-plus-position } \\
\text { of the hand }\end{array}$ & 73.3 & 100 & 78.9 & 19.5 & $<0.01$ & $<0.01$ & $<0.01$ \\
\hline \multicolumn{8}{|c|}{$\begin{array}{l}\text { Responder rates (\%); response was definded as } \geq 1 \text {-point improvement on the Ashworth Scale for all treated muscle groups at study end; Fisher's exact test was } \\
\text { used for group comparisons }\end{array}$} \\
\hline & & & \multicolumn{3}{|c|}{ INCO $n=93$} & & $\operatorname{CON} n=83$ \\
\hline \multicolumn{3}{|c|}{ Ambulatory medical treatment } & \multicolumn{3}{|c|}{175} & & 217 \\
\hline \multicolumn{3}{|l|}{ Drugs } & \multicolumn{3}{|c|}{3,386} & & 193 \\
\hline \multicolumn{3}{|c|}{$\begin{array}{l}\text { Hospitalizations } \\
\text { (including rehabilitation measures) }\end{array}$} & \multicolumn{2}{|c|}{40} & & & 138 \\
\hline \multicolumn{3}{|l|}{$\begin{array}{l}\text { Non-pharmacological } \\
\text { therapies }\end{array}$} & \multicolumn{2}{|c|}{1,408} & & & 998 \\
\hline \multicolumn{3}{|l|}{ Medical devices / aids } & \multicolumn{2}{|c|}{79} & & & 12 \\
\hline \multicolumn{3}{|l|}{ Nursing home care } & \multicolumn{2}{|c|}{3,089} & & & 2,203 \\
\hline \multicolumn{3}{|l|}{ Total direct costs } & \multicolumn{2}{|c|}{8,188} & & & 3,806 \\
\hline \multicolumn{3}{|c|}{ Reduction in earning capacity } & \multicolumn{2}{|c|}{2,081} & & & 988 \\
\hline \multicolumn{3}{|l|}{ Total costs } & \multicolumn{2}{|c|}{10,268} & & & 4,794 \\
\hline
\end{tabular}

Table 6 Overview of Cost-utility ratios and ICER

\begin{tabular}{|c|c|c|c|c|c|}
\hline Utility parameter & INCO & & CON & & \\
\hline Responder rate in Ashworth Score per clinical pattern & Responder rate & Cost-utility ratio & Responder rate & Cost-utility ratio & ICER \\
\hline Shoulder adduction / internal rotation & $62.9 \%$ & $16,325 €$ & $15.5 \%$ & $30,929 €$ & $11,549 €$ \\
\hline Shoulder abduction & $73.0 \%$ & $14,066 €$ & $19.7 \%$ & $24,335 €$ & $10,271 €$ \\
\hline Shoulder elevation & $72.7 \%$ & $14,124 €$ & $20.6 \%$ & $23,272 €$ & $10,507 €$ \\
\hline Flexed elbow & $83.8 \%$ & $12,253 €$ & $26.9 \%$ & $17,821 €$ & $9,621 €$ \\
\hline Pronated forearm & $79.0 \%$ & $12,998 €$ & $22.0 \%$ & $21,791 €$ & $9,604 €$ \\
\hline Flexed wrist & $86.2 \%$ & $11,912 €$ & $26.6 \%$ & $18,022 €$ & $9,185 €$ \\
\hline Thumb-in-palm & $78.8 \%$ & $13,031 €$ & $20.0 \%$ & $23,970 €$ & $9,310 €$ \\
\hline Clenched fist & $84.4 \%$ & $12,166 €$ & $22.2 \%$ & $21,595 €$ & $8,801 €$ \\
\hline Intrinsic-Plus-position (hand) & $78.9 \%$ & $13,014 €$ & $19.5 \%$ & $24,585 €$ & $9,216 €$ \\
\hline Improvement in SF-12 dimension & Improvement & Cost-utility ratio & Improvement & Cost-utility ratio & ICER \\
\hline Physical Health & 7.96 & $1,290 €$ & 0.83 & $5,776 €$ & $768 €$ \\
\hline Mental Health & 10.75 & $955 €$ & 5.71 & $840 €$ & $1,086 €$ \\
\hline
\end{tabular}




\section{Author details}

'Institute of Empirical Health Economics, Am Ziegelfeld 28, D-51399

Burscheid, Germany. ${ }^{2}$ Movement Disorders Section, Department of

Neurology, Hannover Medical School, Hannover, Germany. ${ }^{3}$ Merz

Pharmaceuticals, Frankfurt/M, Germany.

Received: 11 August 2016 Accepted: 11 August 2016

Published online: 31 August 2016

\section{Reference}

1. Rychlik R, Kreimendahl F, Schnur N, Lambert-Baumann J, Dressler D. Quality

of life and costs of spasticity treatment in German stroke patients. Health

Economics Review. 2015;6(1):1-9.

\section{Submit your manuscript to a SpringerOpen ${ }^{\circ}$ journal and benefit from:}

- Convenient online submission

- Rigorous peer review

- Immediate publication on acceptance

- Open access: articles freely available online

- High visibility within the field

- Retaining the copyright to your article

Submit your next manuscript at $>$ springeropen.com 